\title{
Effect of pH conditions on the depolymerization of Wucaiwan coal by mixed acids/ultrasound method and the product structures and performance
}

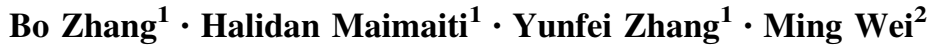

Received: 11 January 2017/Revised: 19 July 2017 / Accepted: 17 October 2017/Published online: 1 November 2017

(C) The Author(s) 2017. This article is an open access publication

\begin{abstract}
The cleavage of the aliphatic chain or ether bond connecting the polycyclic aromatic hydrocarbons in coal can be achieved by not only hydrogenation reduction but also oxidative acid treatment. In this paper, coal samples from Wucaiwan in Xinjiang were pretreated with $\mathrm{HNO}_{3}$ followed by mixed acids/ultrasound treatment. The depolymerized coal samples obtained under different $\mathrm{pH}$ conditions were then separated by fractional washing. The structures and properties of the resulting coal samples were studied by elemental analysis, FT-IR, XRD, TG-DTA, TEM, UV-Vis, and PL. The results showed that when $\mathrm{pH}=0.012$, the obtained coal samples were fragments stripped off from the raw coal samples by ultrasound in strong acid conditions, aliphatic hydrocarbons linked with oxygen-containing groups such as nitro group, a small amount of small aromatic molecules and mineral salts; when $\mathrm{pH}=1.99-4.09$, the obtained coal samples were polycyclic aromatic hydrocarbons linked with oxygen-containing groups such as nitro group,possessing the annulus wall of multilayer graphene fragment structures built up by $\mathrm{sp}^{2}$ carbons, and they are typical fluorescent substances of carbon nanoparticle structure. The former has no solubility in organic solvents, while the latter can be well dissolved in polar solvents such as acetone. All the depolymerized coal samples obtained under different $\mathrm{pH}$ conditions exhibited good absorption and ability of fluorescence emission.
\end{abstract}

Halidan Maimaiti

m15899160730@163.com

1 Institute of Chemistry and Chemical Industry, Xinjiang

University, Urumqi 830046, Xinjiang, China

2 State Key Laboratory of Fine Chemicals, Dalian University of Technology, Dalian 116024, China 


\section{Graphical Abstract}
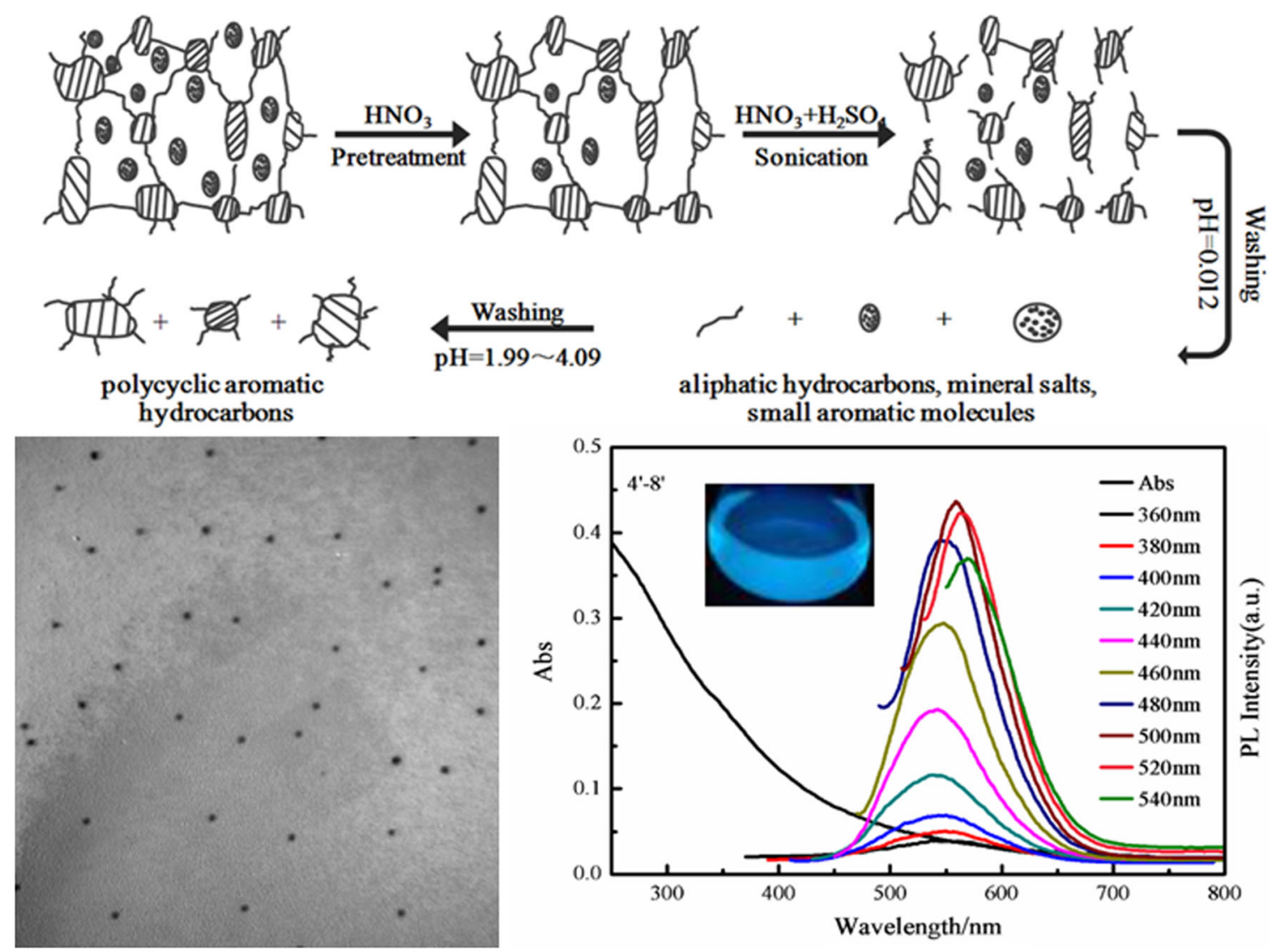

Keywords Coal $\cdot$ Depolymerization $\cdot \mathrm{pH}$ conditions $\cdot$ Carbon dots $\cdot$ Fluorescent

\section{Introduction}

During the coal gasification and liquefaction processes, hydrogen can cleave the alkane chain or ether bond between the macromolecules in coal structures in the presence of catalyst to obtain liquid hydrogenated products (Chen et al. 2015; Li et al. 2015; Guo et al. 2015). The cleavage of these weak bonds can be achieved by not only hydrogenation reduction but also acid oxidation. For instance, Zhao et al. treated Dahuangshan bituminous coal samples with $\mathrm{H}_{2} \mathrm{SO}_{4} / \mathrm{HNO}_{3}$ (3:1) mixed acids and solubilized the treated samples in $\mathrm{N}$, N-dimethylformamide (DMF) and mixed them with polyacrylonitrile (PAN) as spinning solution and produced coal-based carbon fibres by using electrospinning technique (Zhao et al. 2014); Hu et al. used Shanxi bituminous coal as carbon source and treated them with nitric acids and prepared coal-based fluorescent carbon dots ( $\mathrm{Hu}$ et al. 2014). Undoubtedly, treating coal with oxidative acids to destroy the macromolecular structures of coal and break down particular chemical bonds and further prepare coal-based high valueadded products is an effective way of realizing efficient utilization of coal resources (Hu et al. 2016). However, in terms of current research status, the main purpose of acid treatment of coal is to remove the minerals and sulfur in coal and loosen the cross-linked structure of coal so that the conversion processes of coal such as pyrolysis, gasification, liquefaction, and solvent extraction can proceed efficiently (Soneda et al.1998; Li et al. 2004a; Zhang et al. 2007; Izquierdo et al. 2011; Manoj and Narayanan 2013; Li et al. 2004b), and little has been reported on the preparation of specific products from acid treated coal.

Therefore, aiming at preparing coal-based carbon nanoparticles via depolymerizing coal, our research group treated Xinjiang Wucaiwan coal samples with a mixed acids/ultrasound method and found for the first time that varying the system $\mathrm{pH}$ values during the washing step can result in different depolymerization degree, product structure, product dispersity in organic solvents, and optical property of product aqueous dispersion. Our results not 
only presented a method for the preparation of coal-based carbon nanoparticles, but also aid in revealing the coal structures on a molecular level, at the same time, it can provide useful information for the developing technique of mild conversion of coal.

\section{Experimental}

\subsection{Materials and reagents}

Coal sample was collected from Wucaiwan coal mine in Zhundong coal field in Xinjiang, China. It was ground to pass a 200 mesh screen and dried at $105{ }^{\circ} \mathrm{C}$ for $4 \mathrm{~h}$ before use. Dialysis bag (Spectrumlabs, molecular weight cut off 100-500) was purchased from Shanghai Toscience Biotechnology Co., Ltd. $\mathrm{H}_{2} \mathrm{SO}_{4}, \mathrm{HNO}_{3}$, DMSO, DMF, acetone, and chloroform $\left(\mathrm{CHCl}_{3}\right)$ are of analytical grade and commercially available.

\subsection{Depolymerization of coal samples}

A weighed amount of coal sample powder passing through 200 mesh screen was added into $2.6 \mathrm{M}$ of $\mathrm{HNO}_{3}$ aqueous solutions. The mixture was stirred at room temperature for $24 \mathrm{~h}$ and refluxed for another $24 \mathrm{~h}$ and then cooled to room temperature. The remaining solid was separated from the mixture by centrifugation and air dried. A weighed amount of the dried solid was transferred into a flask and soaked in a certain amount of mixed acids of $\mathrm{H}_{2} \mathrm{SO}_{4} / \mathrm{HNO}_{3}$ (3:1 volume ratio) and sonicated at $60{ }^{\circ} \mathrm{C}$ for $12 \mathrm{~h}$. Then the solid/acid mixture was transferred into a centrifuge tube with some distilled water added. Centrifugation was carried out at a rate of 10,000 rpm. The supernatant was labeled as No. 1. Distilled water was added into the centrifuge tube. After the precipitation was well dispersed by shaking the tube by hand, centrifugation was carried out again and the supernatant was labeled as No. 2. Repeat the steps until the precipitation was completely depolymerized. No. 1, 2, and 3 supernatants were transferred into dialysis bags with corresponding number and dialyzed in water for $72 \mathrm{~h}$, during which water was constantly refreshed, until the $\mathrm{pH}$ was neutral. The solutions in the dialysis bags were the depolymerization products of Wucaiwan coal sample under different $\mathrm{pH}$ conditions. They were dried under nitrogen flow and then weighed.

\subsection{Structural characterization}

A variety of instruments were used to characterize three batches of coal samples: the raw coal sample, the coal sample pretreated with $\mathrm{HNO}_{3}$, and the depolymerized coal samples obtained under different $\mathrm{pH}$ conditions. The content of element $\mathrm{C}, \mathrm{H}, \mathrm{O}, \mathrm{N}$, and $\mathrm{S}$ in all three coal samples was determined by FLASHEA-PE2400 elemental analyzer. The ash content in all three samples was estimated by analyzing the weight changes of the samples during the programmed temperature using Kaiyuan 5EMAG6700 industrial analyzer. FT-IR and XRD characterizations of all three samples were performed by BrukerEQUINOX 55 Fourier Transform Infrared Spectrometer and MAC-M18XHF22-SRA X-Ray Diffractometer, respectively. The thermal properties of the three samples were determined by PE-DTA/1700 thermal analyzer. The aqueous dispersions of depolymerized coal samples obtained under different $\mathrm{pH}$ conditions were diluted and dropped onto copper grid and dried to allow observation of the morphology and structure of all sample particles using Japan Hitachi H-600 Transmission Electron Microscope (TEM). The particle sizes of the aqueous dispersion were measured by using NANO- S90laser particle size analyzer.

\subsection{Properties of depolymerized coal samples}

Depolymerized coal samples obtained under different $\mathrm{pH}$ conditions were dispersed in water and then diluted. The aqueous dispersions were transferred into cuvettes and measured by Japan Hitachi UV 3900H UV-Vis spectrophotometer for UV-Vis adsorption spectra and by France Horiba Fluorolog-3 fluorescence spectrometer for emission spectra at different excitation wavelengths, respectively. The solubility of depolymerized coal samples in organic solvents was observed by dispersing the samples into DMSO, DMF, acetone, and $\mathrm{CHCl}_{3}$.

\section{Results and discussion}

\section{1 $\mathrm{HNO}_{3}$ pretreatment of coal samples}

Table 1 lists the pretreatment method for Wucaiwan coal sample, yield, and elemental and ash analysis results of the treated sample.

Elemental analysis results showed that after pretreatment with $\mathrm{HNO}_{3}$, ash in the coal was removed and the content of $\mathrm{S}$ decreased while the content of both $\mathrm{O}$ and $\mathrm{N}$ increased.

To study the structure change of the coal sample before and after the pretreatment by $\mathrm{HNO}_{3}$, FT-IR, XRD, and TGDTG analysis were performed on three samples in Table 1 and the results are shown in Fig. 1, 2.

Figure 1a shows that the FT-IR spectrum (curve 1) of Wucaiwan coal sample is consistent with that of other coal samples (Saikia et al. 2008; Manoj and Elcey 2010; Balachandran 2014). Three types of peaks were found: (1) the peaks at 2923 and $1376 \mathrm{~cm}^{-1}$ correspond to 
Table 1 Preprocessing results of WUCAIWAN coal in $\mathrm{HNO}_{3}$

\begin{tabular}{|c|c|c|c|c|c|c|c|c|}
\hline \multirow[t]{2}{*}{ Sample } & \multirow{2}{*}{$\begin{array}{l}\text { Processing } \\
\text { method }\end{array}$} & \multirow{2}{*}{$\begin{array}{l}\text { Yield }^{\mathrm{a}} \\
(\%)\end{array}$} & \multicolumn{6}{|c|}{ Ultimate analysis (wt\%) } \\
\hline & & & $\mathrm{C}$ & $\mathrm{H}$ & $\mathrm{O}^{\mathrm{b}}$ & $\mathrm{N}$ & $S$ & $\mathrm{~A}_{\mathrm{d}}^{\mathrm{c}}$ \\
\hline 1 & Raw coal & 99.0 & 75.04 & 3.43 & 16.29 & 0.58 & 0.52 & 4.14 \\
\hline 2 & $\begin{array}{l}\text { Sample } 1 \\
\text { mild } \\
\text { stirring in } \\
\mathrm{HNO}_{3} \text { at } \\
25^{\circ} \mathrm{C} \text { for } \\
24 \mathrm{~h}\end{array}$ & 97.3 & 69.56 & 3.18 & 25.07 & 1.11 & 0.24 & 0.84 \\
\hline 3 & $\begin{array}{l}\text { Sample } 2 \\
\text { stirring } \\
\text { for } 24 \mathrm{~h} \\
\text { of reflux }\end{array}$ & 71.1 & 63.65 & 2.61 & 32.12 & 1.39 & 0.23 & 0.0 \\
\hline
\end{tabular}

${ }^{a}$ The ratio of the mass of the dry matter after treatment and the sample before ${ }^{\mathrm{b}} \mathrm{By}$ difference

${ }^{c}$ The $A_{d}$ is ash content, its analysis reference for the Chinese national stan$\operatorname{dard}(\mathrm{GB} / 212-91)$

the aliphatic structure; (2) the peaks at 3050, 1585, 826, and $763 \mathrm{~cm}^{-1}$ are the characteristic peaks of aromatic ring; (3) the peaks at $3350,1715,1250 \mathrm{~cm}^{-1}$ are assigned to the oxygen-containing functional groups. The band between 400 and $600 \mathrm{~cm}^{-1}$ relates to the ash. Curve 2 represents the FT-IR spectrum of the coal sample after $\mathrm{HNO}_{3}$ treatment at $25{ }^{\circ} \mathrm{C}$ for $24 \mathrm{~h}$. The shape of curve 2 is almost the same as that of curve 1 except the decrease of the band between 400 and $600 \mathrm{~cm}^{-1}$ and the increase of the peaks at 1715 and $1250 \mathrm{~cm}^{-1}$, indicating that treating raw coal sample with $\mathrm{HNO}_{3}$ at room temperature aided in the removal of ash and the increase of oxygen-containing functional groups in the structure of coal. Curve 3 is the FT-IR spectrum of the coal sample after $24 \mathrm{~h}$ of reflux. It shows that the intensities of the adsorption at 1715 and $1250 \mathrm{~cm}^{-1}$ further increase for sample 3. Besides that, a weak band appeared between 3000 and $2500 \mathrm{~cm}^{-1}$ and a single peak showed up at $1538 \mathrm{~cm}^{-1}$, corresponding to the adsorption of $\mathrm{COOH}$ and $\mathrm{C}-\mathrm{NO}_{2}$ group, respectively. In addition, both the peak of $\mathrm{O}-\mathrm{H}$ group at $3350 \mathrm{~cm}^{-1}$ and the peak of aliphatic structure at $2923 \mathrm{~cm}^{-1}$ decrease, and the peak at $1376 \mathrm{~cm}^{-1}$ assigned to the aliphatic structure disappeared, and also the band between 400 and $600 \mathrm{~cm}^{-1}$ associated with the ash almost disappeared. The results of ultimate analysis and FT-IR demonstrate that treating Wucaiwan coal sample with $\mathrm{HNO}_{3}$ under reflux condition not only was efficient in removing the ash in the coal but also introduced oxygencontaining groups such as nitro group into the organic structure of coal and reduced the content of aliphatic structure relatively.

Figure $1 \mathrm{~b}$ shows that all three samples exhibited two distinct peaks at $26^{\circ}$ and $43^{\circ}$ in their X-ray diffraction (XRD) pattern, corresponding to the (002) plane of
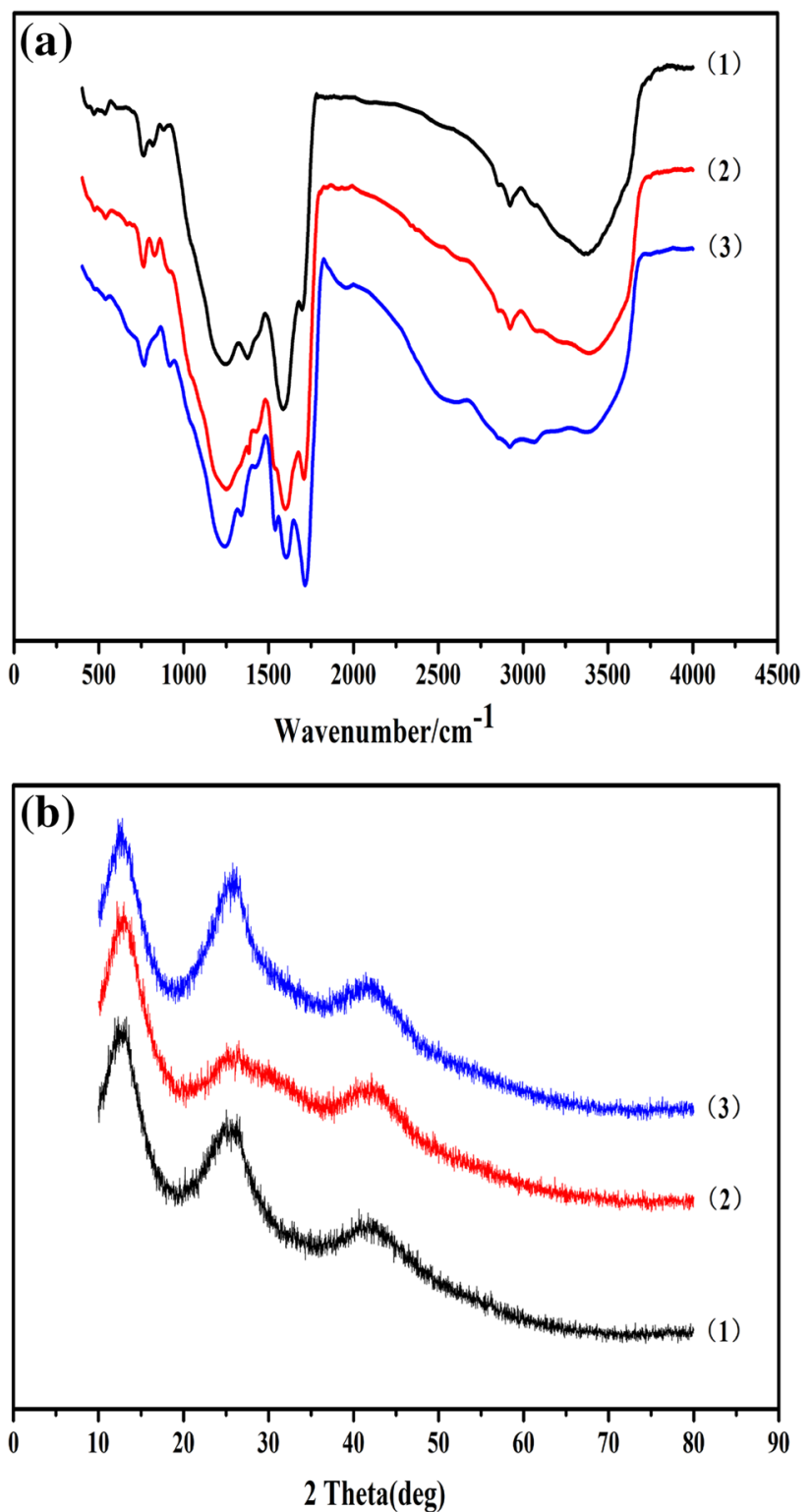

Fig. 1 FT-IR (a) and XRD (b) spectra of coal and its pretreatment (1) coal, (2) Coal mild stirring in $\mathrm{HNO}_{3}$ at room temperature for $24 \mathrm{~h}$, (3) Coal stirring for $24 \mathrm{~h}$ of reflux after mild stirring in $\mathrm{HNO}_{3}$ at room temperature for $24 \mathrm{~h}$

graphite (JCPDS 26-1079) (Hu et al. 2014). After treatment with $\mathrm{HNO}_{3}$, the peak for the (002) plane of sample 2 and 3 slightly shifted to higher degree compared with raw coal sample (sample 1), and gradually approached the (002) plane of graphite at $26.6^{\circ}$, but the peak width and height showed a significant difference, i.e., the peak of sample 2 which was only treated at $25^{\circ} \mathrm{C}$ became wider and shorter while the peak of sample 3 which was treated with $\mathrm{HNO}_{3}$ under reflux became narrower and higher. The results reveal that $\mathrm{HNO}_{3}$ treatment under reflux condition can not only lower the ash content in the coal structure but also reduce the content of aliphatic structure, thereby increasing 

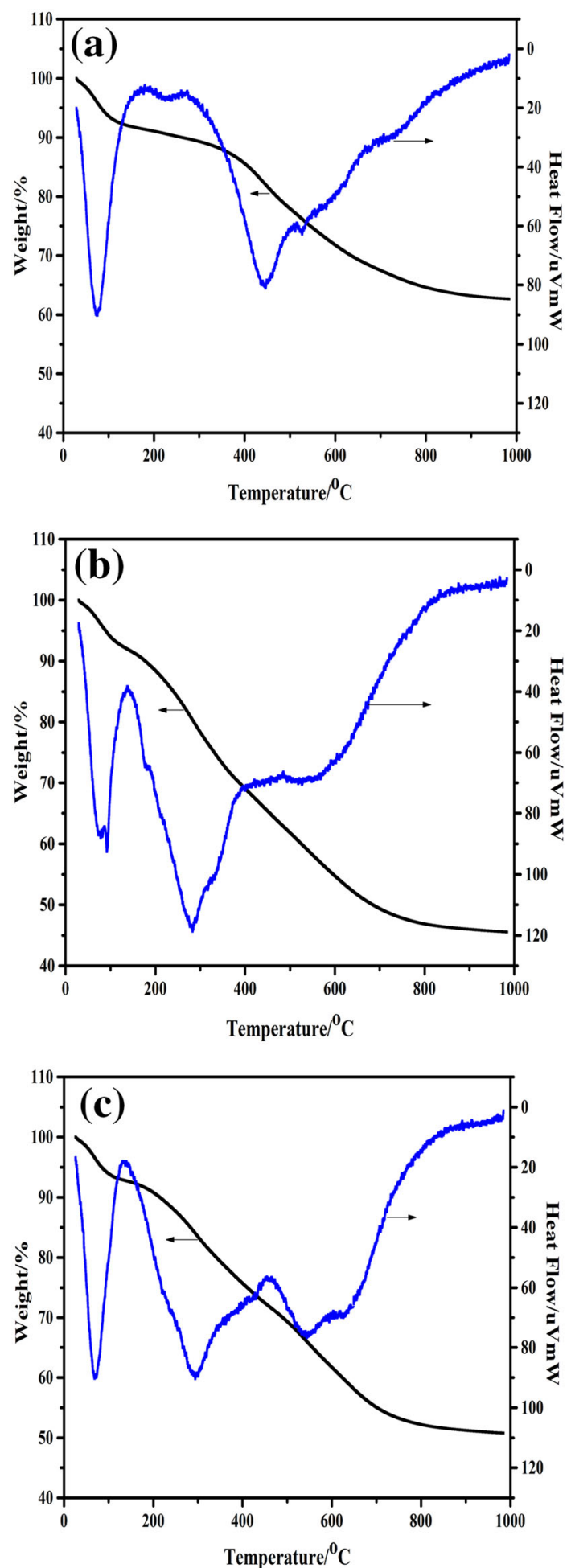

Fig. 2 The TG and DTG curves of coal and its pretreatment a coal, b Coal mild stirring in $\mathrm{HNO}_{3}$ at room temperature for $24 \mathrm{~h}$, c Coal stirring for $24 \mathrm{~h}$ of reflux after mild stirring in $\mathrm{HNO}_{3}$ at room temperature for $24 \mathrm{~h}$

the relative content of polycyclic aromatic rings (Taylor and Bell 1980; Takagi et al. 2004). The XRD results are consistent with the FT-IR results, which may be attributed to the partial breakdown of the aliphatic chains linking the aromatic units by $\mathrm{HNO}_{3}$ under reflux condition.

It has been reported that the weight loss of coal sample under $150{ }^{\circ} \mathrm{C}$ is due to the vaporization of water; the weight loss between 150 and $300{ }^{\circ} \mathrm{C}$ corresponds to the volatilization of light component and the decomposition or dehydration condensation of reactive groups such as carboxyl and hydroxyl groups; the weight loss between 300 and $500{ }^{\circ} \mathrm{C}$ is mainly the pyrolysis of active bridge bonds in the coal, which is a devolatilization process and tar is produced; a second pyrolysis occurs between 600 and $800{ }^{\circ} \mathrm{C}$, which is mainly a process of condensation of aromatic structures and dehydrogenation to produce semicoke (Shi et al. 2013). Therefore, the first weight loss peak at $75{ }^{\circ} \mathrm{C}$ of raw coal sample (Fig. 2a) is generated by the loss of water, and the second peak at $445{ }^{\circ} \mathrm{C}$ is the weight loss peak of devolatilization during pyrolysis. As the temperature increases, especially above $600{ }^{\circ} \mathrm{C}$, polycondensation dominates and the weight loss becomes more gradual.

As seen in Figs. 2b, c, first of all, sample 2 obtained by $\mathrm{HNO}_{3}$ treatment at $25{ }^{\circ} \mathrm{C}$ and sample 3 obtained by $\mathrm{HNO}_{3}$ treatment under reflux condition, both showed peaks of loss of water at 82 and $69^{\circ} \mathrm{C}$, which is similar to the behavior of raw coal sample. Second, sample 2 and 3 showed a major weight loss peak at 284 and $293{ }^{\circ} \mathrm{C}$, respectively, corresponding to the volatilization of small molecular compounds and the decomposition or dehydration condensation of oxygen-containing groups such as carboxyl and hydroxyl groups, this is distinctly different from the weight loss behavior of raw coal sample, it indicates that by treatment with oxidative $\mathrm{HNO}_{3}$, a large number of active oxygen-containing functional groups appeared in the coal structure. Noted that within this range sample 3 has lower temperatures for water loss peak and decomposition peak of oxygen-containing groups than that of sample 2, demonstrating that the content of oxygen-containing groups in sample 3 is higher than that in sample 2, because the existent of oxygen-containing groups can not only facilitate the decomposition and dehydration condensation of coal but also induce the pyrolysis of organic matter and decrease the pyrolysis temperature (Fletcher et al. 2007; Ruiz et al. 2006). Moreover, the oxygen-containing groups in sample 2 and 3 were basically removed by the first pyrolysis, resulting in higher dehydrogenation condensation coking temperature than that of raw coal sample during the second pyrolysis; therefore, both sample 2 and 3 exhibited weight loss peaks between 500 and $600{ }^{\circ} \mathrm{C}$. In addition, the weight loss ratio of sample 2 within the $0-1000{ }^{\circ} \mathrm{C}$ temperature range is higher than that of sample 3 , which is mainly caused by initial loss of water. 


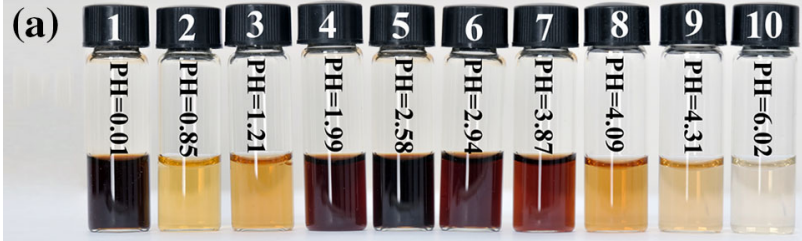

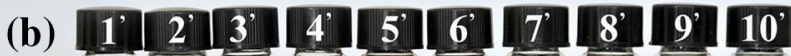

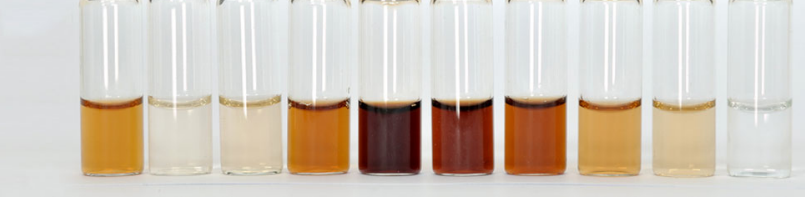

Fig. 3 Photos of depolymerization products of coal in water medium under visible light. a Containing acid; $\mathbf{b}$ as been to remove acid by dialysis

\subsection{Depolymerization of pretreated coal samples by mixed acids/ultrasound}

A certain proportion of mixed acids $\mathrm{H}_{2} \mathrm{SO}_{4} / \mathrm{HNO}_{3}$ (3:1 volume ratio) were added into the coal sample pretreated with $\mathrm{HNO}_{3}$ and the mixture was sonicated at $60{ }^{\circ} \mathrm{C}$ for $12 \mathrm{~h}$. After sonication, the acid/coal mixture was washed by purified water for several times. Figure 3 are the photos of the washing solution obtained by washing the above sample (first treated with $\mathrm{HNO}_{3}$ and then by mixed acids/ ultrasound for $12 \mathrm{~h}$ ) with $100 \mathrm{~mL}$ of purified water for 10 times (a), and the aqueous dispersion of the depolymerized coal samples after acid removal by dialysis (b). Both photos were taken under visible light. It can be inferred by the color of the washing solution in Fig. 3 that when washing the mixture to different $\mathrm{pH}$ conditions, the amount and structure of depolymerized coal samples dispersing in water may be different.

FT-IR, fluorescent spectroscopy, and particle size distribution measurement, etc., were performed on group (b) aqueous dispersions with darker colors $\left(1^{\prime}, 4^{\prime}-8^{\prime}\right)$. The results showed that samples $4^{\prime}-8^{\prime}$ had similar particle sizes and structures, and also their luminescence behaviors were basically the same, while the FT-IR spectrum, the particle size, and the luminescence behavior of sample $1^{\prime}$ were distinctly different from that of samples $4^{\prime}-8^{\prime}$. Therefore, we grouped samples $4^{\prime}-8^{\prime}$ for analysis and compared the results with sample $1^{\prime}$.

Table 2 lists the yield of the water soluble depolymerized coal samples (labeled as sample 4 and 5) obtained by air drying sample $1^{\prime}$ and the collected solution of samples $4^{\prime}-8^{\prime}$ and their elemental analysis results.

The results in Table 2 showed that $56.1 \%$ of the coal sample pretreated with $\mathrm{HNO}_{3}$ and sonicated in mixed acids for $12 \mathrm{~h}$ depolymerized when washed for the first time $(\mathrm{pH}$ of the washing solution was 0.012). However, after dialysis, the color of the washing solution became significantly lighter, and the yield of the water soluble product obtained by air drying of the washing solution was quite low, only $18.7 \%$. The elemental analysis results indicated that the carbon content of this depolymerized coal sample was relatively low, meaning that $81.3 \%$ of the depolymerized product of the coal samples obtained under strong acid condition are low-molecular-weight fluidic substance, or short chain small molecules and carbonaceous gas obtained from disruption of the aliphatic structures by strong acid. With the increase of the washing time, the system $\mathrm{pH}$ gradually increased, and the depolymerization degree of the coal sample changed from almost zero at $\mathrm{pH}=0.85-1.21$ to substantial depolymerization at $\mathrm{pH}=1.99-4.09$, and the amount of the product to remove by dialysis was not much, the yield of the water soluble product reached $64.6 \%$ (the color of the washing solution obtained at this $\mathrm{pH}$ turned slightly lighter, but it was caused by the penetration of water into the dialysis bag diluting the solution during the dialysis process). Moreover, the content of carbon in the product increased, indicating the content of aromatic structures in the depolymerized coal samples obtained at $\mathrm{pH}=1.99-4.09$ is relatively high.

Table 2 The results of mixed acid/ultrasonic processing

\begin{tabular}{|c|c|c|c|c|c|c|c|c|c|}
\hline \multirow[t]{2}{*}{ Sample } & \multirow[t]{2}{*}{ Processing method } & \multirow[t]{2}{*}{$\mathrm{pH}$ of washing solution ${ }^{\mathrm{a}}$} & \multirow[t]{2}{*}{ Depolymerization rate $^{\mathrm{b}}(\%)$} & \multirow[t]{2}{*}{$\operatorname{yield}^{\mathrm{c}}(\%)$} & \multicolumn{5}{|c|}{ Ultimate analysis (wt\%) } \\
\hline & & & & & $\mathrm{C}$ & $\mathrm{H}$ & $\mathrm{O}^{\mathrm{d}}$ & $\mathrm{N}$ & $S$ \\
\hline 4 & $1^{\prime}$ dry matter & 0.012 & 56.1 & 18.7 & 43.59 & 3.45 & 44.54 & 2.78 & 5.64 \\
\hline 5 & $4^{\prime}-8^{\prime}$ dry matter & $1.99-4.09$ & 80.1 & 64.6 & 53.46 & 1.31 & 42.60 & 2.11 & 0.52 \\
\hline
\end{tabular}

${ }^{\mathrm{a}} \mathrm{The} \mathrm{pH}$ of washing solution before dialysis

${ }^{\mathrm{b}}$ The ratio of the mass difference of the didn't depolymerization samples before and after mixed acid/ultrasonic treatment and before

${ }^{c}$ The ratio of the mass of the dry matter after dialysis and the sample before mixed acid/ultrasonic treatment

${ }^{\mathrm{d}}$ By difference 

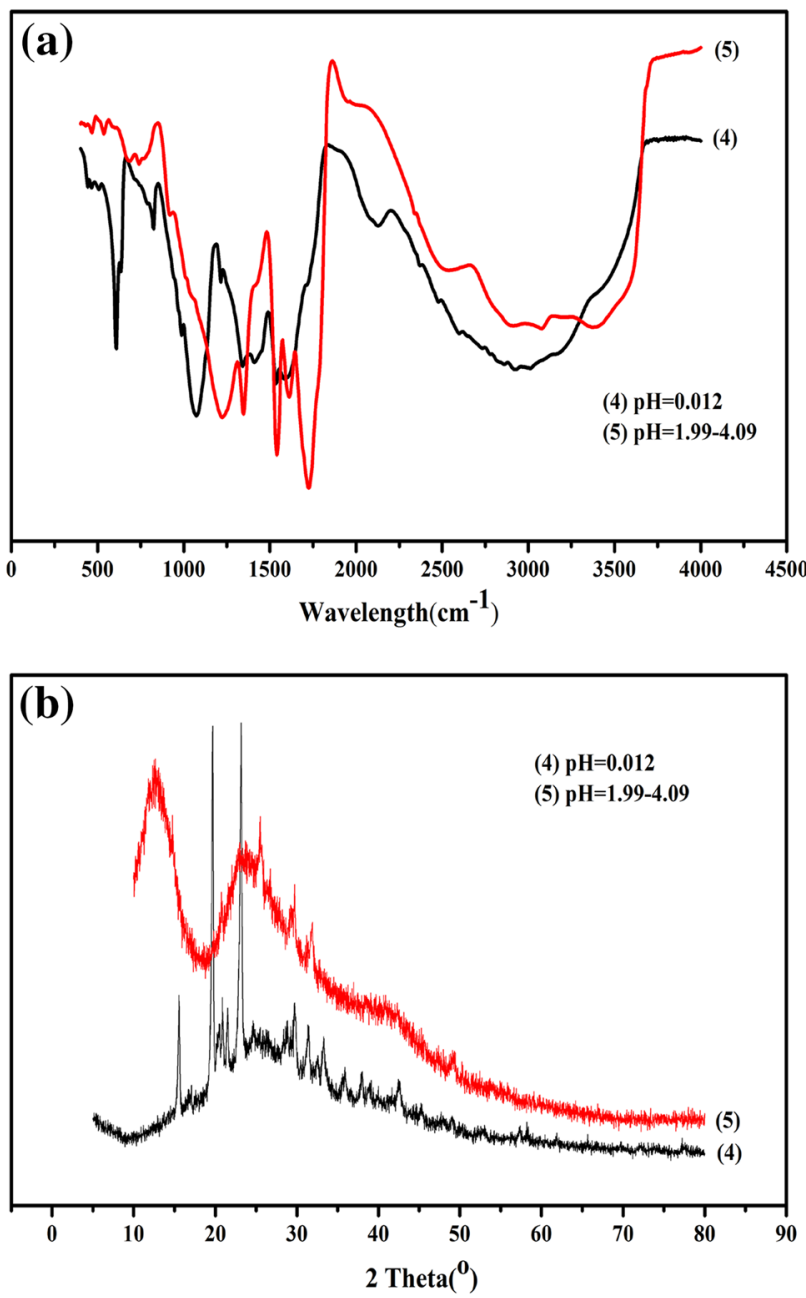

Fig. 4 The FT-IR (a) and XRD (b) spectra of depolymerization products of coal at different $\mathrm{pH}$

\subsection{Structures of depolymerized coal samples obtained under different $\mathrm{pH}$ conditions}

To identify the structures of the depolymerized coal samples obtained under different $\mathrm{pH}$ conditions, we analyzed the two samples in Table 2 by means of FT-IR, XRD, TGDTG, and TEM, etc., and the results are shown in Figs. 4, 5 and 6.

It can be seen from Fig. 4a that the depolymerized product obtained under the strongest acid condition (curve 4) showed characteristic peaks of several functional groups besides the peaks at $3050,1587,824$, and $779 \mathrm{~cm}^{-1}$ corresponding to the adsorption band of aromatic structure: the peak at $2921 \mathrm{~cm}^{-1}$ corresponds to the adsorption band of aliphatic structure; the peaks at 1536, 1341, 634, and $607 \mathrm{~cm}^{-1}$ are attributed to the bending vibration bands of $\mathrm{C}-\mathrm{NO}_{2}$ and $\mathrm{C}-\mathrm{N}=\mathrm{O}$ groups and cis- and trans- $\mathrm{O}-\mathrm{N}=\mathrm{O}$ group that link the aromatic compounds; the adsorption band of $\mathrm{COOH}$ group appeared between 3000 and
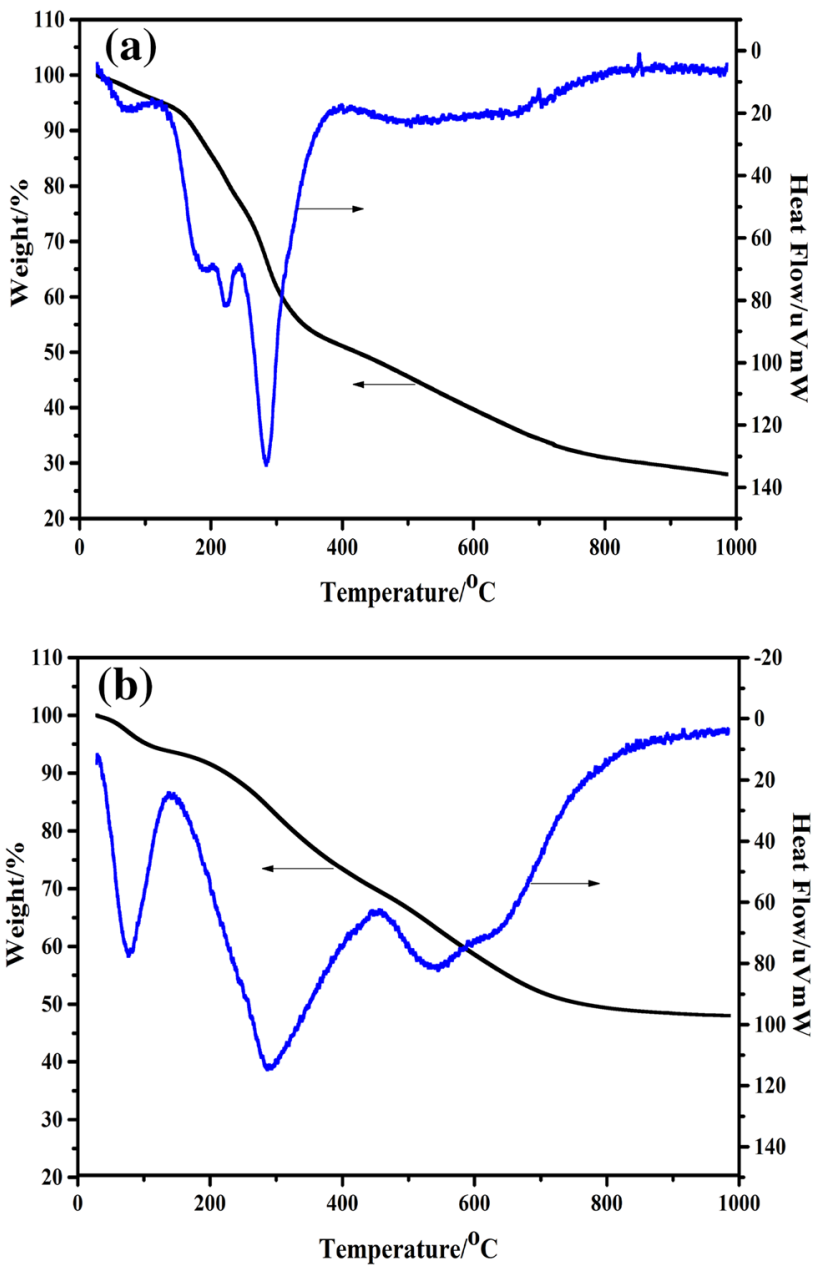

Fig. 5 TG and DTG curves of depolymerization products of coal at different $\mathrm{pH}$ a $\mathrm{pH}=0.012 ; \mathbf{b} \mathrm{pH}=1.99-4.09$

$2100 \mathrm{~cm}^{-1}$; the two peaks at 1214 and $1073 \mathrm{~cm}^{-1}$ are indicative of the $\mathrm{C}-\mathrm{O}-\mathrm{C}$ unsymmetric and symmetric stretching vibration band; the shoulder at $1720 \mathrm{~cm}^{-1}$ is associated with the adsorption band of $\mathrm{C}=\mathrm{O}$ group; the peak at $1410 \mathrm{~cm}^{-1}$ corresponds to the adsorption band of the sulfate group $\mathrm{R}-\mathrm{O}-\mathrm{SO}_{2}-\mathrm{OR}^{\prime}$; the adsorption band of $\mathrm{Si}-\mathrm{O}$ group showed up as a strong peak at $1073 \mathrm{~cm}^{-1}$; the bands at $1000-900$ and $506-424 \mathrm{~cm}^{-1}$ are assigned to the adsorption band of $\mathrm{SO}_{4}{ }^{2-}$. These results reveal that the depolymerized product obtained under the strongest acid condition contains not only aromatic compounds linked with oxygen-containing groups including carboxyl, nitro, carbonyl, and sulfate groups and aliphatic compounds, but also mineral salts such as sulfate salts and silicon oxides which may be produced by the reaction of the minerals in the coal structure with the mixed acids. Curve 5 is the FTIR spectrum of the depolymerized product obtained at $\mathrm{pH}=1.99-4.09$, the characteristic peaks of which are summarized: the peaks at 3076 and $740 \mathrm{~cm}^{-1}$ correspond to the adsorption band of the $-\mathrm{CH}$ group in aromatic 

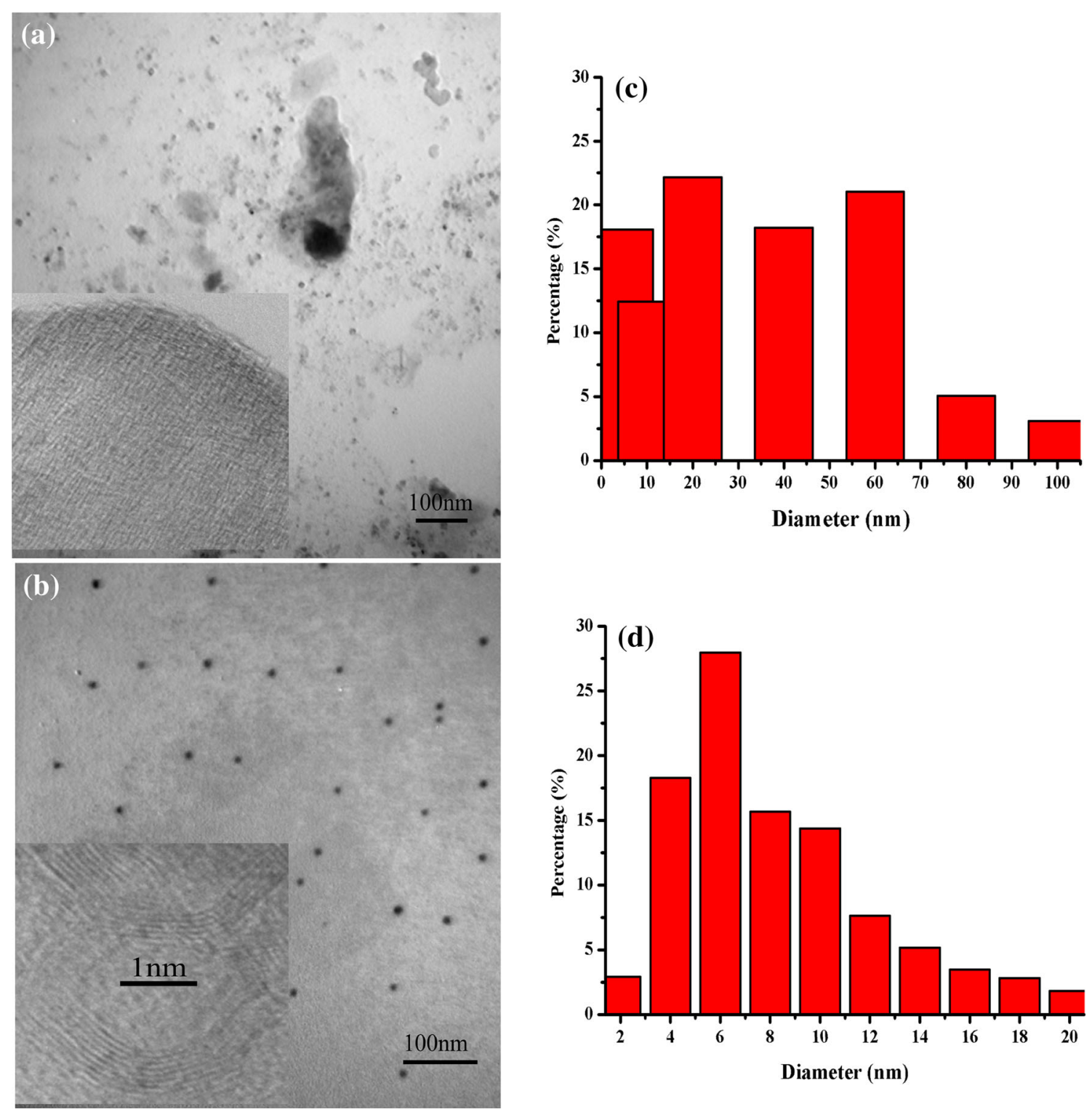

Fig. 6 TEM micrographs $(\mathbf{a}, \mathbf{b})$ and particle size distribution $(\mathbf{c}, \mathbf{d})$ of depolymerization products of coal at different $\mathrm{pH}: \mathbf{a}, \mathbf{c} \mathrm{pH}=0.012 \mathbf{b}$, d $\mathrm{pH}=1.99-4.09$, Illustrations for high power TEM

compounds; the in-plane bending vibration band of the $\mathrm{CH}$ group in aromatic ring appeared at $1223 \mathrm{~cm}^{-1}$; the adsorption band of $\mathrm{COOH}$ group showed up between 3000 and $2300 \mathrm{~cm}^{-1}$; the peak at $3371 \mathrm{~cm}^{-1}$ is assigned to the adsorption band of $-\mathrm{OH}$ group; the peak at $1725 \mathrm{~cm}^{-1}$ is the characteristic peak of the carbonyl group $\mathrm{C}=\mathrm{O}$; the peak at $1612 \mathrm{~cm}^{-1}$ is attributed to the adsorption band of aryl ketone group; the two peaks at 1538 and $1345 \mathrm{~cm}^{-1}$ correspond to the unsymmetric and symmetric stretching vibration band of the nitro group linking the aromatic compounds, respectively; the peaks at 667 and $600 \mathrm{~cm}^{-1}$ are associated with the bending vibration band of the $\mathrm{O}-$ $\mathrm{N}=\mathrm{O}$ group in aromatic compounds; the adsorption band of sulfur containing groups appeared at 909, 536, and
$467 \mathrm{~cm}^{-1}$. The results indicate that the depolymerized product obtained at $\mathrm{pH}=1.99-4.09$ is aromatic compound linked with more oxygen-containing groups including hydroxyl, carboxyl, ester, carbonyl, nitro, and sulfonic groups.

It can be seen from Fig. $4 \mathrm{~b}$ that both samples exhibited diffraction peaks between $15^{\circ}$ and $40^{\circ}$ corresponding to the (002) plane of graphite, among which the peak of sample 4 is wider with a lot of unidentified peaks, indicating that the content of aliphatic structure and minerals in the depolymerized product obtained at $\mathrm{pH}=0.012$ is high (Taylor and Bell 1980; Takagi et al. 2004). However, the (002) plane of sample 5 is higher and narrower than that of sample 4 and most of the unidentified peaks disappeared, 
indicating that the content of aromatic structure in the depolymerized product obtained at $\mathrm{pH}=1.99-4.09$ increases, and the molecular orientation between aromatic planes is more ordered, i.e., the coal structure is gradually graphitized (Zou et al. 2008).

The weight losses of the depolymerized coal samples obtained at $\mathrm{pH}=0.012$ and $\mathrm{pH}=1.99-4.09$ at $1000{ }^{\circ} \mathrm{C}$ are $72.1 \%$ and $52.0 \%$, respectively, as shown in Fig. 5, and weight loss of the latter was largely caused by the loss of water at low temperature, indicating that the depolymerized coal samples obtained at $\mathrm{pH}=1.99-4.09$ are structurally more stable. In addition, the maximum weight loss of sample 4 (Fig. 5a) basically occurred in the temperature range below $290{ }^{\circ} \mathrm{C}$, i.e., when the temperature was above $290{ }^{\circ} \mathrm{C}$, the curve smoothed and the weight loss ratio lowered. According to the statement of the reference mentioned above (Shi et al. 2013), weight loss below $290{ }^{\circ} \mathrm{C}$ corresponds to the volatilization of small molecular compounds and the decomposition or dehydration condensation of oxygen-containing groups such as carboxyl and hydroxyl groups, demonstrating that the depolymerized coal samples obtained under the strongest acid condition mainly consist of small molecular substances linked with oxygen-containing groups, and the content of aromatic structure is relatively low. For sample 5 (Fig. 5b) besides the weight loss peak at $291{ }^{\circ} \mathrm{C}$ indicative of the decomposition or condensation of the oxygen-containing groups such as carboxyl and hydroxyl groups, the weight loss peak appeared corresponding to the condensation of aromatic structure and the production of semicoke by dehydrogenation between 500 and $600{ }^{\circ} \mathrm{C}$, indicating again that the depolymerized coal samples obtained at $\mathrm{pH}=1.99-4.09$ are mostly aromatic substance linked with oxygen-containing groups.

Particles of all sizes and shapes were obtained after dialysis of the depolymerized coal samples obtained under strong acid condition $(\mathrm{pH}=0.012)$ to remove acid and low-molecular-weight fluidic substance (Fig. 6a) and the particle size distribution (Fig. 6c) is between 1 and $100 \mathrm{~nm}$. It can be seen from its High Resolution TEM (HRTEM) images that the majority of the depolymerized coal sample particles obtained under strong acid condition possess irregular structures similar to that of amorphous carbon.

Sphere particles with uniform shapes were obtained after dialysis of the depolymerized coal samples obtained at $\mathrm{pH}=1.99-4.09$ (Fig. $6 \mathrm{~b}$ ) and the percentage of the particles with a size distribution (Fig. 6d) between 2 and $10 \mathrm{~nm}$ is as high as $79.15 \%$. HRTEM images further show that these sphere particles are annulations, the annulus walls of which have lattice fringes similar to graphite structure (see inset), and the lattice spacing is $0.32 \mathrm{~nm}$ and
$0.21 \mathrm{~nm}$, corresponding to the (002) and (100) plane of graphite, respectively (Zhao et al. 2008).

Coal is a natural polymer. The two-phase model proposed by Marzec et al. stated that there are a large number of polycyclic aromatic hydrocarbons in coal molecules which are linked by aliphatic chains or ether bonds, forming the macromolecular phase of the coal molecules; the second phase is composed of smaller aromatic molecules which are distributed between the gaps of the first phase (Marzec 1986). This is extremely similar to the coal structure model raised by Shinn in 1986 through the products from the first and second stage liquefaction of coal (Shinn 1984). This type of structure explains the phenomena of organic solvents extracting small molecules from coal and the phenomena of coal swelling in certain organic solvents, thus it is the most authoritative theory for coal structure. Taking the two-phase model as the initial coal structure studied in this paper and combining the results from FT-IR, XRD, TG-DTG, TEM and particle size distribution analysis, we consider that the process of depolymerization of coal under the action of mixed acid and ultrasound as Fig. 7.

Under the action of mixed acid and ultrasound, the raw coal sample was shattered and the aliphatic structures or ether bonds of coal was disrupted at first. Then, the aliphatic hydrocarbons linked with oxygen-containing groups such as nitro group, mineral salts, and small aromatic molecules distributed between the gaps of the first phase was dissolved in water phase when washing the system to $\mathrm{pH}=0.012$. As the washing number increase $(\mathrm{pH}=1.99-4.09)$, the rest of the sediment depolymerized, and the depolymerized coal samples are composed of only aromatic compound linked with more oxygen-containing groups including hydroxyl, carboxyl, ester, carbonyl, nitro, and sulfonic groups, possessing the annulus wall of multilayer graphene fragment structures built up by $\mathrm{sp}^{2}$ carbons, as the polycyclic aromatic hydrocarbons lost aliphatic chains or ether bonds is easier to oxidation when the proportion of mixed acid and the water reached to a certain value (Ross et al. 1986). They are typical fluorescent substances of carbon nanoparticle structure (Eda et al. 2010).

\subsection{Properties of depolymerized coal samples}

Figure 8 shows the absorption and fluorescence emission spectra for sample $1^{\prime}$ (Fig. 8a) and the collected solutions of samples $4^{\prime}-8^{\prime}$ (Fig. 8b).It can be seen from Fig. 8 that the depolymerized coal samples obtained by mixed acids/ ultrasound treatment all exhibited good absorption and emission ability.

The UV-Vis absorbance spectra show that sample $1^{\prime}$ has broad absorption bands in the ultraviolet-visible light 


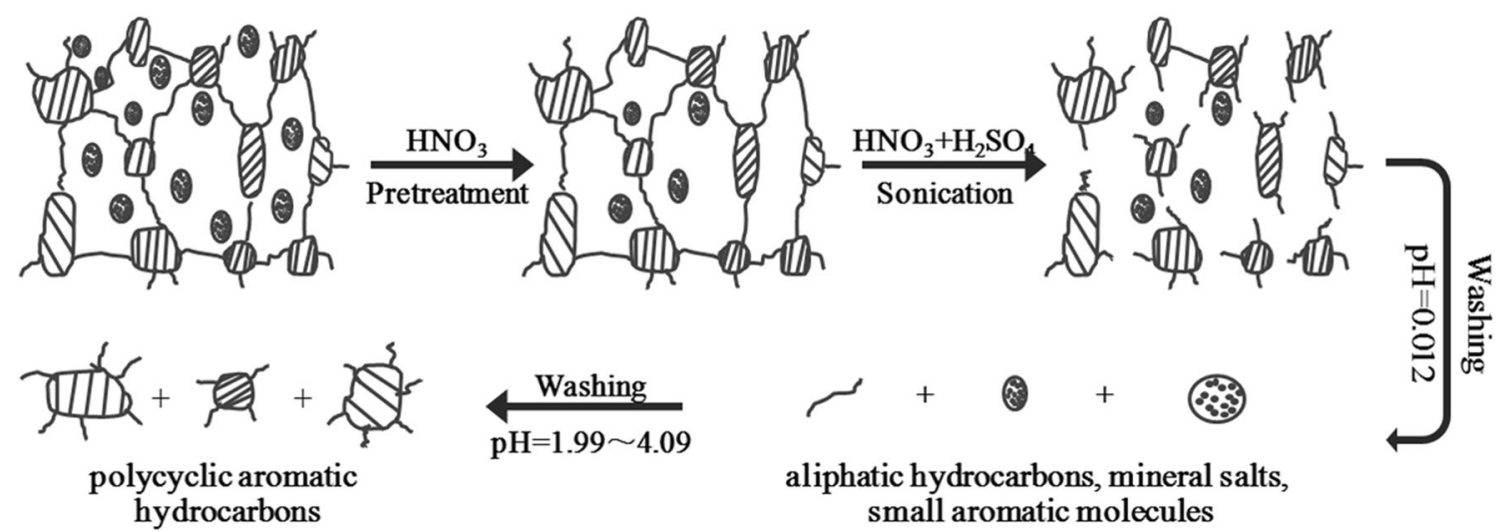

Fig. 7 Schematic diagram of depolymerization processes of the coal samples by oxidative acid treatment
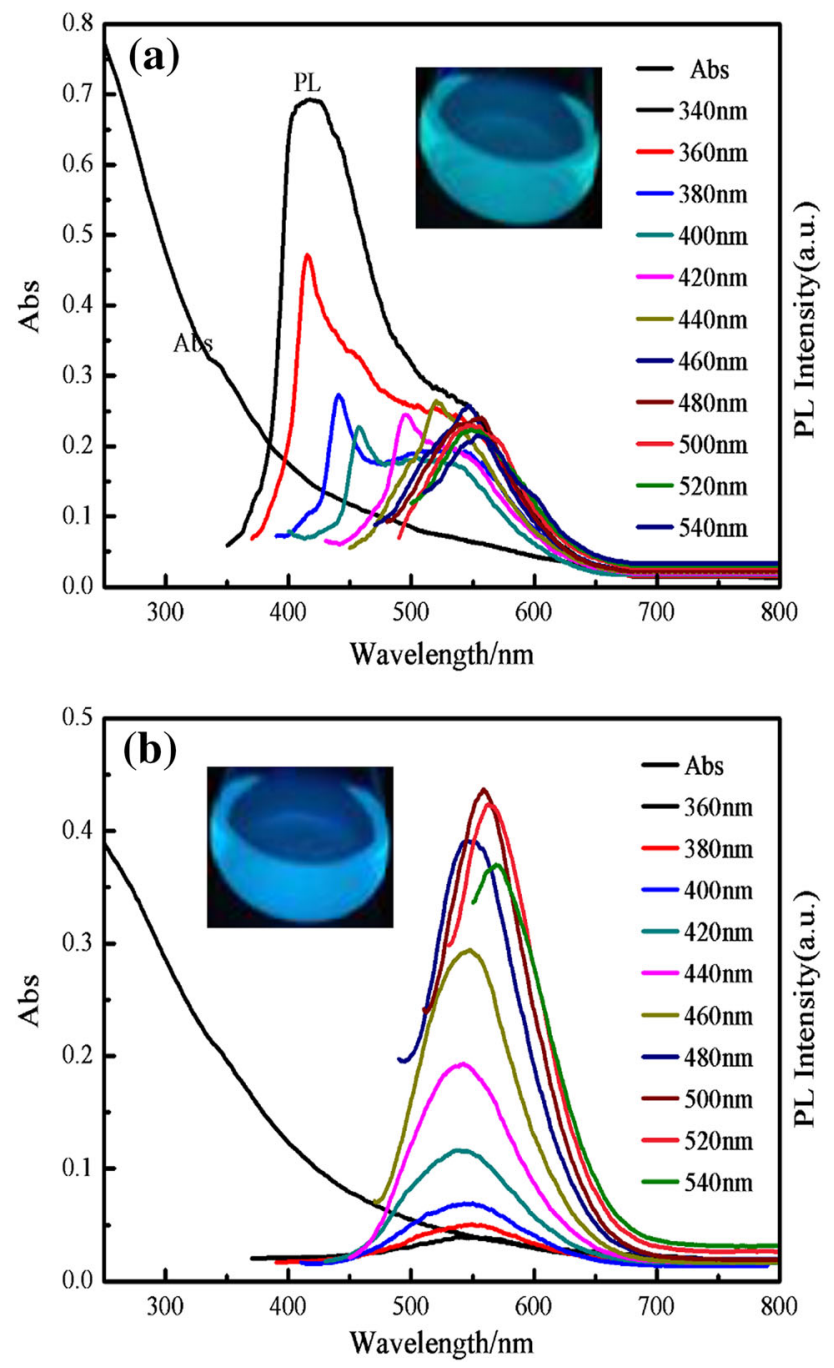

Fig. 8 UV-Vis absorption spectrum and photoluminescent spectra of depolymerization products of coal at different $\mathrm{pH}$ a $\mathrm{pH}=0.012$, b $\mathrm{pH}=1.99-4.09$

region with a shoulder appearing at $340 \mathrm{~nm}$, which may be related to the incorporation of aliphatic structures and small aromatic molecules linked with oxygen-containing groups
Table 3 Solubility of depolymerization products of coal

\begin{tabular}{|c|c|c|c|c|}
\hline \multirow{2}{*}{ Sample } & \multicolumn{4}{|c|}{ Solubility } \\
\hline & DMSO & DMF & ACETONE & $\mathrm{CHCl}_{3}$ \\
\hline 4 & + & + & + & - \\
\hline 5 & - & - & - & - \\
\hline
\end{tabular}

such as nitro group, and complex compositions including raw coal fragments and minerals in sample $1^{\prime}$, as well as the uneven distributions of particle sizes. When sample $1^{\prime}$ was excited by the light of 340-440 nm wavelength, two maxima showed up in its fluorescence emission spectrum. The first one is between 416 and $506 \mathrm{~nm}$, the peak shifted to longer wavelength and the intensity decreased gradually as the excitation wavelength increased. The second emission peak is located at around $550 \mathrm{~nm}$, and the maximum wavelength did not shift with the change of the excitation wavelength, but the peak intensity first increased then decreased. When the excitation wavelength is longer than $440 \mathrm{~nm}$, the fluorescence emission peak became a single one, red shift occurred with the increase of the excitation wavelength, and the peak intensity first increased then decreased. The optimal excitation wavelength reached at around $500 \mathrm{~nm}$, where the strongest emission peak appeared at around $550 \mathrm{~nm}$.

The collected solution of samples $4^{\prime}-8^{\prime}$ contains mainly polycyclic aromatic hydrocarbons with large $\pi$ bonds, and they are linked with more heteroatom chromophores including $\mathrm{C}=\mathrm{O}, \mathrm{C}=\mathrm{N}, \mathrm{C}=\mathrm{S}, \mathrm{N}=\mathrm{N}, \mathrm{N}=\mathrm{O}$, and $-\mathrm{NO}_{2}$, etc., which may result in $\pi \rightarrow \pi^{*}$ and $\mathrm{n} \rightarrow \pi^{*}$ transitions simultaneously (Shull 1964); therefore, they have broad adsorption bands in the ultraviolet and visible light region. When the collected solution of samples $4^{\prime}-8^{\prime}$ was excited with the light of $360-540 \mathrm{~nm}$ wavelength, there was a 
single peak in the fluorescence emission spectrum, the intensity of which first increased then decreased, red shift occurred with the increase of the excitation wavelength, and the optimal excitation wavelength reached at around $520 \mathrm{~nm}$, where the strongest emission peak appeared at around $560 \mathrm{~nm}$. The fluorescence property is basically the same as other typical luminescent carbon nanoparticles, depending on excitation wavelengths and having multiple excitation and multiple emission (Hana et al. 2009; Zhu et al. 2012; Liu et al. 2012).

In addition, upon exposure to the light of $365 \mathrm{~nm}$ wavelength from portable UV lamp, sample $1^{\prime}$ exhibited blue-green fluorescence and the collected solution of samples $4^{\prime}-8^{\prime}$ exhibited blue fluorescence (inset in Fig. 8). Meanwhile, we used quinine sulfate as reference and measured the fluorescence quantum yields of sample $1^{\prime}$ and the collected solution of samples $4^{\prime}-8^{\prime}$ to be 6.79 and $17.32 \%$, respectively, based on the reference method. $17.32 \%$ is higher than the quantum yield of any other coalbased carbon dots ever reported (Hu et al. 2014).

The solubility of the depolymerized coal samples obtained at $\mathrm{pH}=0.012$ (sample 4) and $\mathrm{pH}=1.99-4.09$ (sample 5) in DMSO, DMF, acetone, and $\mathrm{CHCl}_{3}$ were measured as shown in Table 3.

Coal molecules are composed of large aromatic building blocks linked by aliphatic chains or ether bonds, thus coal is considered cross-linked polymer, which can only swell in organic solvents but cannot dissolve (Bazylyak et al. 2007). From the structural characterization results above, we know that the coal structure has undergone a fundamental change after the mixed acids/ultrasound treatment. First of all, under the action of ultrasound in mixed acids, the ether bonds in the structure of $\mathrm{HNO}_{3}$ pretreated coal sample may be protonated and ruptured by the attack from nucleophiles such as nitro group. Second, strong oxidative mixed acids may directly destroy the aliphatic chains that link the aromatic building blocks with the help of ultrasound, making the aromatic building blocks no longer cross-linked and separate from each other. Third, oxidative groups may be produced on the edge of the aromatic building blocks due to the strong oxidative effect on the aromatic hydrocarbons in the coal structure. The subsequence of the changes above apparently can make the coal sample depolymerize and dissolve in organic solvents. However, in terms of the experimental results, only the depolymerized coal samples obtained at $\mathrm{pH}=1.99-4.09$ have good solubility in DMSO, DMF and acetone, and the depolymerized coal samples obtained at $\mathrm{pH}=0.012$ can't dissolve in those solvent (Table 3). Therefore, taking the characterization results of the depolymerized coal samples above into consideration, it was concluded that only when the $\mathrm{pH}$ of the coal-acid system was $1.99-4.09$ can the oxygen-containing aromatic building blocks in the treated coal samples be disintegrated into independent aromatic units, while when the $\mathrm{pH}$ of the coal-acid system was 0.012 , the obtained depolymerized coal samples mainly contain destroyed small molecular aliphatic chains, coal fragments and minerals.

In addition, oxygen-containing groups make the aromatic fragments very polar; therefore, the depolymerized coal samples obtained at $\mathrm{pH}=1.99-4.09$ can be well dissolved in polar solvents such as DMSO, DMF and acetone, but cannot be dissolved in low polarity solvents such as $\mathrm{CHCl}_{3}$.

\section{Conclusions}

Under the action of mixed acid and ultrasound, $56.1 \%$ of the coal samples pretreated with $\mathrm{HNO}_{3}$ depolymerized $(\mathrm{pH}$ of the coal-acid system was 0.012 ), among which $81.3 \%$ of the depolymerized coal samples are low-molecular-weight fluidic substances, or short chain small molecules and carbonaceous gas obtained from disruption of the aliphatic structures by strong acid, and $18.7 \%$ are aliphatic compounds linked with oxygen-containing groups including carboxyl, nitro, carbonyl, sulfate groups and small aromatic molecules, and minerals such as sulfate salts and silicon oxides. Continue to wash the sediment haven't depolymerization to $\mathrm{pH}=0.85-1.21$, it basically did not depolymerize; when washing to $\mathrm{pH}=1.99-4.09$, a large number of the coal samples depolymerized, and the yield for the water soluble substances reached $64.6 \%$. it is found by analysis that the depolymerized coal samples obtained at $\mathrm{pH}=1.99-4.09$ have single component consisting of polycyclic aromatic hydrocarbons linked with oxygencontaining groups such as nitro group, can be well dissolved in polar solvents such as acetone, and its aqueous solution exhibited good absorption and ability of fluorescence emission, they are typical graphitized fluorescent substances of carbon nanoparticle structure.

Through the study of the paper, we not only confirmed the structure model of coal put forward by Marzec, but also believe that it is feasible treating coal with oxidative acids to destroy the macromolecular structures of coal and break down particular chemical bonds and further prepare coalbased high value-added products.

Open Access This article is distributed under the terms of the Creative Commons Attribution 4.0 International License (http://crea tivecommons.org/licenses/by/4.0/), which permits unrestricted use, distribution, and reproduction in any medium, provided you give appropriate credit to the original author(s) and the source, provide a link to the Creative Commons license, and indicate if changes were made. 


\section{References}

Balachandran M (2014) Role of infrared spectroscopy in coal analysis - an investigation. Am J Anal Chem 5:367-372

Bazylyak LI, Bryk DV, Makitra RG, Prystansky RE, Zaikov GE (2007) Generalisation of data concerning coal swelling in organic solvents and their extraction using linear multiparametric equations. J Balk Tribol Assoc 13:182-196

Chen B, Diao ZJ, Zhao YL, Ma XX (2015) A ReaxFF molecular dynamics (MD) simulation for the hydrogenation reaction with coal related model compounds. Fuel 154:114-122

Eda G, Lin Y, Mattevi C, Yamaguchi H, Chen HA, Chen IS, Chen CW, Chhowalla M (2010) Blue photoluminescence from chemically derived graphene oxide. Adv Mater 22:505-509

Fletcher AJ, Uygur Y, Thomas KM (2007) Role of surface functional groups in the adsorption kinetics of water vapor on microporous activated carbons. J Phys Chem C 111:8349-8359

Guo BF, Zhou W, Liu S, Liu Y, Wang J, Yin R (2015) Effect of $\gamma$-ray irradiation on the structure and electrochemical liquefaction of Shenhua coal. Fuel 143:236-243

Hana Ž, Církva V, Hájek M (2009) Microwave synthesis of fluorescent carbon nanoparticles with electrochemiluminescence properties. Chem Commun 103:5118-5120

Hu C, Yu C, Li MY, Wang X, Yang J, Zhao Z, Eychmüller A, Sun YP, Qiu J (2014) Chemically tailoring coal to fluorescent carbon dots with tuned size and their capacity for $\mathrm{Cu}(\mathrm{II})$ detection. Small 10:4926-4933

Hu SL, Wei ZJ, Chang Q, Trinchi A, Yang J (2016) A facile and green method towards coal-based fluorescent carbon dots with photocatalytic activity. Appl Surf Sci 378:402-407

Izquierdo MT, Rubio B, Yuso AMD, Ballestero D (2011) Enhancement of nitric oxide removal by ammonia on a low-rank coal based carbon by sulphuric acid treatment. Fuel Process Technol 92:1362-1367

Li C, Takanohashi AT, Saito I, Aoki H, Mashimo K (2004a) Elucidation of mechanisms involved in acid pretreatment and thermal extraction during ashless coal production. Energy Fuels 18:97-101

Li C, Takanohashi T, Yoshida T, Ikuo S, Aoki H, Mashimo K (2004b) Effect of acid treatment on thermal extraction yield in ashless coal production. Fuel 83:727-732

Li XH, Xue YL, Feng J, Yi Q, Li W, Guo X, Liu K (2015) Copyrolysis of lignite and Shendong coal direct liquefaction residue. Fuel 144:342-348

Liu S, Tian J, Wang L, Zhang Y, Qin X, Luo Y, Asiri AM, Al-Youbi AO, Sun X (2012) Hydrothermal treatment of grass: a low-cost green route to nitrogen-doped carbon-rich photoluminescent polymer nanodots as an effective fluorescent sensing platform for label-free detection of $\mathrm{Cu}(\mathrm{II})$ ions. Adv Mater 24:2037-2041

Manoj B, Elcey CD (2010) Demineralization of coal by stepwise bioleaching: a study of sub-bituminous Indian coal by FTIR and SEM. J Univ Chem Technol Metall 45:385-390
Manoj B, Narayanan P (2013) Study of changes to the organic functional groups of a high volatile bituminous coal during organic acid treatment process by FTIR spectroscopy. J Miner Mater Charact Eng 01:39-43

Marzec A (1986) Macromolecular and molecular model of coal structure. Fuel Process Technol 14:39-46

Ross DS, Gu CL, Hum GP, Malhotra R (1986) Oxidation of benzyl alcohols by nitrous and nitric acids in strong sulfuric acid media. Int J Chem Kinet 18:1277-1288

Ruiz B, Parra JB, Pajares JA, Pis JJ (2006) Effect of coal preoxidation on the optical texture and porosity of pyrolysis chars. J Anal Appl Pyrol 75:27-32

Saikia BK, Boruah RK, Gogoi PK (2008) FT-IR and XRD analysis of coal from Makum coalfield of Assam. J Earth Syst Sci 116:575-579

Shi L, Liu QY, Guo XJ, Wu W, Liu Z (2013) Pyrolysis behavior and bonding information of coal—a TGA study. Fuel Process Technol 108:125-132

Shinn JH (1984) From coal to single-stage and two-stage products: a reactive model of coal structure. Fuel 63:1187-1196

Shull H (1964) Electronic Spectra and quantum chemistry. J Am chem Soc 26:5371-5372

Soneda Y, Makino M, Yasuda H, Yamada O, Kobayashi M, Kaiho M (1998) The effect of acid treatment of coal on $\mathrm{H}_{2} \mathrm{~S}$ evolution during pyrolysis in hydrogen. Fuel 77:907-911

Takagi H, Maruyama K, Yoshizawa N, Yamada Y, Sato Y (2004) $\mathrm{XRD}$ analysis of carbon stacking structure in coal during heat treatment. Fuel 83:2427-2433

Taylor ND, Bell AT (1980) Effects of Lewis acid catalysts on the cleavage of aliphatic and aryl-aryl linkages in coal-related structures. Fuel 59:499-506

Zhang B, Wu D, Wang C, He S, Zhang Z, Kong H (2007) Simultaneous removal of ammonium and phosphate by zeolite synthesized from coal fly ash as influenced by acid treatment. J Environ Sci 19:540-545

Zhao QL, Zhang ZL, Huang BH, Peng J, Zhang M, Pang DW (2008) Facile preparation of low cytotoxicity fluorescent carbon nanocrystals by electrooxidation of graphite. Chem Commun 41:5116-5118

Zhao HY, Wang LX, Jia DZ, Xia W, Li J, Guo ZP (2014) Coal based activated carbon nanofibers prepared by electrospinning. J Mater Chem 2:9338-9344

Zhu CZ, Zhai JF, Dong SJ (2012) Bifunctional fluorescent carbon nanodots: green synthesis via soy milk and application as metalfree electrocatalysts for oxygen reduction. Chem Commun 48:9367-9369

Zou L, Kang FY, Li XL, Zheng YP, Shen W, Zhang J (2008) Investigations on the modified natural graphite as anode materials in lithium ion battery. J Phys Chem Solids 69:1265-1271 